\title{
Adolescence and mental health
}

Sarah-Jayne Blakemore

Adolescence is a time hallmarked by change: changes to hormones and the body, changes in the social environment, and changes to the brain and the mind. Although most young people develop into healthy adults, the period of adolescence confers significant vulnerability to mental health problems. Many mental illnesses - including depression, anxiety, eating disorders, substance abuse disorders and psychosis - first appear before the age of 24 years. What is it about adolescence that increases vulnerability to mental health problems? This is a crucial question as many mental illnesses that start in adolescence persist throughout adult life, creating long term morbidity and a substantial burden on society. Depression alone is estimated to become the world's leading cause of disability-adjusted life years by 2030.

Adolescence is defined as the period of life between puberty and adult independence, which depends on both individual development and cultural norms. Based on the large cultural differences in social expectations of this age group, some have argued that the concept of adolescence is a twentieth century, Western invention. I would argue that, on the contrary, adolescence should be considered a distinct period of biological, psychological and social development for three main reasons. First, adolescent-typical behaviours, such as heightened risk-taking and sensation seeking, can be seen across different human cultures. Second, there is evidence of adolescent-typical behaviour across species. All mammals undergo a stage of development between puberty and becoming sexually mature adults, and during that stage there is evidence of increased risk-taking, exploration of the environment and changes in social behaviour. Third, adolescent-typical behaviours are well documented across history. More than 2000 years ago, Socrates (469-399 BC) wrote: 'They have bad manners, contempt for authority; they show disrespect for elders and love chatter in place of exercise.' A hundred years or so later, Aristotle described 'youth' as 'lacking in sexual self-restraint, fickle in their desires, passionate and impulsive... Youth is the age when people are most devoted to their friends', highlighting the notion that adolescence is a period of fluctuating and intense emotions, in addition to the changing social landscape of this time of life. Adolescents have for millennia been portrayed in many of the - largely negative - ways they are today.

Adolescence starts with significant changes in hormones and the body, and this coincides with a sharp increase in mental health problems. Puberty is characterised by rapid increases in oestrogen in girls and testosterone in boys, and this is when sex differences in mental health problems start to emerge. For example, before puberty depression occurs at around the same, relatively low rate in girls and boys, whereas after puberty, there is a sharp increase in the incidence of depression, with adolescent girls around twice as likely to experience it as boys. Starting puberty earlier than one's peers is a risk factor for depressive symptoms and anxiety, especially in girls. Adolescent boys, in contrast, are more likely than girls to develop substance use disorders and to die by suicide.

At the same time as the body is changing, so is the brain. In the past two decades, large-scale, longitudinal studies using magnetic resonance imaging (MRI) have revealed that the brain undergoes substantial and protracted development throughout human adolescence and into adulthood. Different types of brain tissue in different brain regions show varying patterns of maturational development. White matter volume increases across the brain during adolescence and into adulthood. Conversely, the volume of cortical grey matter is highest in late childhood and decreases substantially - by approximately $1.5 \%$ each year according to recent studies - during adolescence, before stabilising in the mid-twenties. Regions of the brain involved in perception and movement (sensory and motor cortices) mature earlier than other regions such as the prefrontal, parietal and temporal cortices, which are involved in higher level cognitive processes and continue to develop into the twenties or thirties.

A number of neurodevelopmental processes are thought to be associated with the changes in grey and white matter. These include axonal myelination and increases in axon diameter, changes to the structural properties of brain cells that increase the transmission speed of neuronal signals between different brain regions. A third process involves reorganisation of synapses. During early development, a vastly excess number of synapses is produced, most of which appear to be unspecified for function. Which synapses remain and which are eliminated depends at least in part on environmental experience, in that synapses 
that are not used are eliminated in a process called synaptic pruning. Histological studies of post-mortem brain tissue of different ages have shown that the number of synapses in the human prefrontal cortex is highest in late childhood and then decreases throughout the second and third decades until the number stabilises. These neurodevelopmental processes - myelination of axons, axonal growth and synaptic reorganisation - constitute mechanisms of neuroplasticity, by which brain development is influenced by environmental experience. It is possible that this renders the developing adolescent brain particularly vulnerable to stressful or negative environmental experiences.

Adverse childhood experiences such as abuse, neglect or being the victim of bullying at school are associated with later mental health problems. Other environmental factors that have been found to increase the risk of mental illness (in particular psychosis) include consumption of cannabis during the teenage years, being an immigrant in a culture with different sociocultural values, and growing up in urban, as opposed to rural, environments. What makes some people vulnerable to these risks and others resilient is not well understood. One of the largest risk factors for adolescent mental health problems is parental mental illness. However, while genetic factors are undoubtedly a key mechanism of risk transmission for mental health problems, the social environment also plays an important role and, furthermore, nature and nurture do not work in isolation. For example, a negative family environment might amplify genetic risk factors for mental illness, and genetic factors can influence both the social experiences we seek out during adolescence, as well as how those around us treat us.

Adolescence is naturally characterised by large changes in the social environment that are usually adaptive and positive, but which can be aversive or stressful. There are effects resulting from the physical maturational changes of puberty, which alter the way a young person perceives themselves, as well as the way society perceives and treats them. A young person who has gone through puberty looks more adult and is often treated as such by the people around them, for example by being given more autonomy, responsibility and culpability. This can act as a new pressure, especially in those who are relatively young when they go through puberty. At around the time of puberty, children in many countries move from being amongst the oldest year group in relatively small primary schools, to being amongst the youngest in larger secondary schools. These schools have more people, with large and unstable social hierarchies. The psychologist Stephanie Burnett Heyes at the University of Birmingham, UK, has shown that social networks tend to be chaotic in early adolescence and become more stable and reciprocal later in the teenage years. During adolescence, time spent with friends increases, compared with in childhood, and adolescents' evaluation of their social and personal worth is more dependent on the views of their peers.

Adolescents are more likely to take risks and experiment when they are with peers than when they are alone. Research from my group has shown that, when it comes to evaluating risks, young adolescents are more likely to be influenced by the opinions of other teenagers than adults. Kate Mills, a developmental neuroscientist at the University of Oregon, and I have proposed that peer influence is heightened because, compared with other age groups, adolescents are more negatively affected by being excluded by their peer group. This hypersensitivity to social exclusion might lead adolescents to make decisions to avoid 'social risk' (the risk of social rejection), even if it means opting to take health and legal risks. It has been proposed that this hypersensitivity to the threat of social rejection might be a risk factor for some mental illnesses such as depression.

At the same time that the brain, the body and the social environment are undergoing substantial change, so is the mind. During childhood and adolescence, several aspects of cognitive ability are changing. Mentalising, the ability to understand other people's minds, and the ability to take another person's perspective, are still developing. Cognitive skills such as planning, inhibiting inappropriate behaviour, and certain forms of memory also continue to improve during adolescence. The ability to consider the future and to delay gratification, as well as one's awareness of the self, become increasingly sophisticated. These cognitive advances provide adolescents with the cognitive machinery to reflect on themselves, what others think about them and their futures. This is a necessary part of becoming an independent adult, but it also places new pressures on the developing person. 
Adolescence is a unique stage of development, when our bodies, hormones and social environment are rapidly changing, our brains are developing and our cognitive capacities become more sophisticated. These changes might render adolescence a period of vulnerability to mental ill health. Most of our knowledge about adolescent development comes from research involving young people in high income countries. Little is known about this unique period of development in the large numbers of young people who grow up in low- and middle-income countries, often in extremely challenging circumstances. Adolescent risk taking and mental health problems are global issues and it is vital to understand more about adolescent development across cultures. Our rapidly expanding understanding of the psychological, social and biological changes in adolescence now needs to be converted into interventions and treatments that aim to support the mental health of young people across the world, and to prevent mental illness before it starts.

\section{FURTHER READING}

Allen NB \& Badcock PB. (2003). The social risk hypothesis of depressed mood: evolutionary, psychosocial, and neurobiological perspectives. Psychological Bulletin. 129(6):887-913.

Arseneault L. (2018). The persistent and pervasive impact of being bullied in childhood and adolescence: implications for policy and practice. Journal of Child Psychology and Psychiatry 59(4):405-421.

Blakemore, S-J. (2018). Avoiding social risk in adolescence. Current Directions in Psychological Science. $27(2), 116-122$.

Blakemore, S-J., \& Mills, K.L. (2014). Is adolescence a sensitive period for sociocultural processing? Annual Review of Psychology, 65, 187-207.

Burnett Heyes, S., Rong-Jih, Y., Block, P., Hiu, C. F., Holmes, E. A. \& Lau, J. Y. F. (2015). Relationship reciprocation modulates resource allocation in adolescent social networks: Developmental effects. Child Development 86(5): 1489-1506.

Foulkes, L. \& Blakemore, S-J. (2018). Studying individual differences in human adolescent brain development. Nature Neuroscience, 21(3), 315-323.

Sawyer SM, Azzopardi PS, Wickremarathne D \& Patton GC. (2018). The age of adolescence. Lancet Child and Adolescent Health. 2(3):223-228.

\section{Acknowledgements}

The author receives funding from the Wellcome, Jacobs Foundation, UKRI GCRF and the MRC. The author is grateful to J. Roiser, T. Dalgleish, E. Kilford, M. Bloomfield, N. Boyce, L. Arseneault, G. Lewis and K. Sleeman for their comments on earlier versions of this essay. 